\title{
HIGHLIGHTS
}

SYSTEMS NEUROSCIENCE

\section{Grin and bear it}

When confronted with a stressful situation, different people have different ways to cope. Some of us might try to free ourselves from the nasty stimulus, whereas others might simply grin and bear it. What is the neurobiological explanation for such variability? A recent paper by Giorgi et al. provides an intriguing lead, identifying differences in the mesocortical dopamine system that correlate with the way in which two different rat strains cope with anxiety-generating stimuli.

Roman high- and low-avoidance rat - RHA/Verh and RLA/Verh, respectively - were selectively bred decades ago to show diametrically different responses to stressful stimuli. When facing an aversive stimulus, RHA/Verh rats avoid it faster and are more active than RLA/Verh rats, which tend instead to show fearrelated behaviours such as freezing. Giorgi et al. challenged these two with three anxiety-generating stimuli - a tail pinch, and the drugs pentylenetetrazol and ZK93426. They measured the release of dopamine in the prefrontal cortex (the mesocortical dopamine system) and in the shell of the nucleus accumbens (the mesolimbic dopamine system), and correlated these measures with the behavioural response of the rats to the anxiogenic stimuli.

Their results showed that the three stimuli increased dopamine release by the mesocortical pathway, in the RHA/Verh rats, but not in the
RLA/Verh rats. Behaviourally, as expected, the anxiety-generating stimuli elicited an increased frequency of freezing episodes in the RLA/Verh rats, but not in the other. In fact, RHA/Verh rats proactively tried to escape from the tail pinch, as evidenced by the increased biting of the tail clamp that was used in this particular experiment.

So, in general terms, the data point to a correlation between the activity of the mesocortical system and the ability of the rats to proactivley cope and gain control over stressful stimuli. This observation is in line with data from primates, indicating a role for the mesocortical dopamine system on cognitive and attentional processes. By contrast, the activity of the mesocortical dopamine system did not correlate with the expression of fear-related behaviours, indicating that this ascending system might not be involved with the emotional aspects of stress. However, we need many additional experiments to go beyond this correlation and establish a true causal relationship between the output of the dopamine pathways and the behavioural response to stress.

Juan Carlos López

\section{(4) References and links} ORIGINAL RESEARCH PAPER Giorgi, O. et al. Dissociation between mesocortical dopamine release and fear-related behaviours in two psychogenetically selected lines of rats that differ in coping strategies to aversive conditions. Eur. J. Neurosci. 17, 2716-2726 (2003)

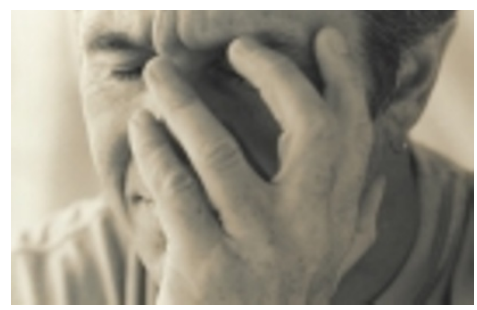

